\title{
Prognostic role of filgrastim in the effectiveness of PD-1/PD-L1 inhibitors in previously treated patients with non-small cell lung cancer
}

JUAN FRANCISCO MARIN POZO ( $\nabla$ jfmarinpozo@yahoo.es )

Complejo Hospitalario de Jaén https://orcid.org/0000-0002-6090-8905

CARMEN LUCIA MUÑOZ CID

Complejo Hospitalario de Jaen

RAQUEL CLARAMUNT GARCIA

Hospital Virgen de Altagracia

ELVIRA MARIN CABA

Hospital Universitario San Cecilio

ANA LAURA ORTEGA GRANADOS

Complejo Hospitalario de Jaen

Research Article

Keywords: nivolumab, pembrolizumab, atezolizumab, filgrastim, real world data

Posted Date: January 25th, 2022

DOI: https://doi.org/10.21203/rs.3.rs-1224958/v1

License: (a) This work is licensed under a Creative Commons Attribution 4.0 International License.

Read Full License 


\section{Abstract}

Purpose: To determine whether the use of G-CSF during the first-line therapy of nSCLC influences the effectiveness of anti-PD1/PD-L1 drugs (aPD1-i) used in subsequent therapies.

Methods: We designed a single-center, observational, retrospective study with nSCLC patients treated with aPD1-i (nivolumab, pembrolizumab, or atezolizumab) after primary platinum-based chemotherapy in southern Spain. The primary endpoints were overall survival (OS) and progression free survival (PFS). The results were assessed using the Kaplan-Meier method for G-CSF-use.

Results: We enrolled 79 patients (91\% male) with a mean age of 66.7 years. Nivolumab, atezolizumab, and pembrolizumab were used in $55.7 \%, 25.3 \%$, and $19.0 \%$ of the patients, respectively. G-CSF was administered to 19 patients. The median treatment duration was 4.1 months ( $95 \% \mathrm{Cl}: 2.3-5.8)$. Overall survival (OS) and progression free survival (PFS) were 7.3 (95\% Cl: $3.4-11.3)$ and 4.4 months (95\% Cl: $2.3-$ 6.5), respectively. The median OS was 15.8 months (95\% Cl: $1.2-30.3)$ in G-CSF-treated patients and 5.3 months $(95 \% \mathrm{Cl}: 2.3-8.3)$ in the non-treated group $(\mathrm{p}=0.186)$. Higher OS was observed in all subgroups.

Conclusions: The effectiveness of aPD1-i in previously treated nSCLC patients was higher in the G-CSFtreated group than in the non-treated group, although the difference was statistically insignificant. These results may contribute to improving the effectiveness of anti-PD1/PD-L1 drugs, although confirmatory prospective studies are required.

\section{Introduction}

Anti-programmed death-1 (PD-1)/programmed death ligand-1 (PD-L1) drugs (aPD1-i) are commonly used for the treatment of non-small-cell lung cancer (nSCLC) (1). aPD1-i are monoclonal antibodies that prevent the binding of PD-1 receptor to the T cells and the binding of PD-L1 to the tumor cells leading to the reversal of $T$ cell inactivation due to this binding, and the antitumor immunity of the T cells is reactivated (2). Monotherapy with nivolumab (NIV), pembrolizumab (PEM), and atezolizumab (ATE) is used in patients with nSCLC who have undergone first-line therapy for nSCLC and have shown an increase in their survival rate, regardless of the degree of expression of PD-1/PD-L1 (3), (4), (5), (6).

The prognostic factors for response are influenced by the characteristics of the patient, tumor, other drugs administered, and environmental factors (7). The tumor-dependent factors, including PD-L1 expression, microsatellite instability (iMS/dMMR), tumor mutational burden (TBM), immunophenotype, and tumorinfiltrating lymphocytes (TILs), are the most studied prognostic factors (8).

Among the patient-dependent factors, the response of the immune system to the tumor is related to the effectiveness of the treatment. It has been observed that the preoperative absolute lymphocyte count (ALC) and absolute monocyte count (AMC) affect disease progression and survival after complete surgical resection of advanced malignant melanoma (9). Similarly, the preoperative peripheral AMC is an important prognostic factor for patients with lung adenocarcinoma after curative resection (10). It has 
been established that the neutrophil/lymphocyte ratio (NLR) is a marker of the general immune response to various stimuli (11). Peripheral neutrophil count measured by NLR is directly correlated with the intratumor neutrophil population (12). Several studies have demonstrated that NLR predicts outcomes in patients with different types of solid cancers (13), (14), (15), (16).

Studies have also correlated NLR with other predictive biomarkers of outcomes in immune checkpoint blockade therapy. In advanced melanoma, increased leukocyte, neutrophil, and monocyte counts, as well as high NLR, have been associated with poor prognosis in patients, especially in the patients receiving ipilimumab (17) or nivolumab (18). In nSCLC, a relationship has been established between high values of NLR or dNLR and poor prognosis of immunotherapy. In a meta-analysis of OS and PFS of 23 studies with 2,068 patients, an elevated NLR predicted poor OS (hazard ratio, HR $=1.62 ; 95 \%$ confidence interval, $95 \% \mathrm{Cl}: 1.41-1.87 ; \mathrm{p}<0.001)$ and PFS (HR $=1.47 ; 95 \% \mathrm{Cl}: 1.25-1.72 ; \mathrm{p}<0.001)(19)$. An NLR $>5$ or a dNLR $>3$ has been proposed as a prognostic factor for the non-effectiveness of nivolumab in nSCLC (20), (21).

Neutropenia is an adverse effect of the chemotherapy regimens employed for the first-line treatment of nSCLC. Treatment of neutropenia is performed using granulocyte colony-stimulating factors (22). Filgrastim (G-CSF) is a colony-stimulating factor that act on hematopoietic cells by binding to specific cell surface receptors and regulating the production of neutrophils within the bone marrow and affects neutrophil progenitor proliferation, differentiation, and selected end-cell functions. G-CSF increases the absolute neutrophil count in patients with neutropenia. Thus, it can be speculated that the use of G-CSF can modify the response of aPD1-i in patients who progress to the initial treatment of nSCLC.

The aim of this study was to determine whether the use of G-CSF during the first-line therapy of nSCLC has an influence on the effectiveness of aPD1-i used in subsequent lines of therapy.

\section{Materials And Methods}

\section{Study Design}

This was an open, observational, retrospective, single-center study conducted at a regional hospital of the Spanish National Health System in southern Spain. All treatment decisions were followed according to the clinical practice protocols used in the center for treatment of nSCLC and chemotherapy-induced neutropenia. We collected data from routine clinical practice, real world data.

The study was conducted in accordance with the Declaration of Helsinki and Good Clinical Practice Guidelines. The protocol was approved by the institutional review board of the hospital.

\section{Participants and variables}

All patients receiving aPD1-i therapy with nivolumab, pembrolizumab or atezolizumab for advanced metastatic nSCLC after initial therapy with platinum doublets between January 2016 and November 2019 
at the study hospital were included. Only patients receiving aPD1-i as part of another clinical trial were excluded.

The primary outcome variables of the study included OS duration (from the start of the aPD1-i therapy to death or censoring) and PFS duration (from the start of the initial aPD1-i therapy to the first recorded occurrence of physician-assessed disease progression or death).

The outcome predictor variables were the use of G-CSF prior to aPD1-i therapy, NLR, and dNLR. Descriptive variables of the population and outcome were also collected, including age, sex, histology, Eastern Cooperative Oncology Group (ECOG) performance-status, aPD1-i used, absolute count of leukocytes (WBC), neutrophils (ANC), and lymphocytes (ALC) prior to treatment, reasons for discontinuation of aPD1-i therapy, and time elapsed between the initiation of initial platinum-derived therapy and the aPD1-i therapy (TiPt).

NLR) and dNLR were calculated as follows:

NLR $=$ ANC/ALC, cut-off $\geq 5$

$\mathrm{dNLR}=\mathrm{ANC} /($ WBC- ANC), cut-off $\geq 3$.

All study variables were collected from the available hospital records, including oncology pharmacy application and medical history, as well as other complementary sources (pathology, laboratory, and radiology). All data were introduced into an ad hoc database.

\section{Statistical Analysis}

The mean, standard deviation, median, range, and counts and percentages (categorical data) were calculated for demographic and cancer characteristics of the patients. PFS and OS were expressed as median survival, with $95 \% \mathrm{Cl}$ using the Kaplan-Meier method to estimate survival curves, and the log-rank test was used to compare the curves. Cox proportional hazards modeling was used to calculate HR and 95\% Cl. Statistical analyses were performed using G-Stat 2.0 (Dep. Biometría GSK. Madrid).

\section{Results}

Seventy-nine patients with nSCLC, who had received treatment, were included in this study; the median age of the patients was 66.7 years $(S D=8.7)$ and $91.1 \%$ were male. Sixty-two patients $(78.5 \%)$ had an ECOG performance-status score of 0 or 1 , and the remaining patients had an ECOG performance-status score of $2(13.9 \%)$. Forty patients $(50.6 \%)$ had squamous tumor histology, and 39 patients $(49.4 \%)$ had non-squamous tumor histology. The following aPD1-i drugs were used in this study: nivolumab in 44 patients (55.7\%), atezolizumab in 20 patients (25.3\%), and pembrolizumab in 15 patients (19.0\%). Treatment with aPD1-i started after a mean duration of 9.7 months $(S D=8.6)$ from the beginning of the first-line platinum-based therapy. G-CSF was administered to 19 patients $(24.1 \%)$. The mean WBC of the patients at the start of the aPD1-i therapy was 9,300 cells $/ \mu \mathrm{L}$ (standard deviation, $\mathrm{SD}=3,900$; range 
3,600-25,800 cells $/ \mu L$ ). NLR was $>5$ in 46 patients (58.2\%), and dNLR was $>3$ in 51 patients $(64.6 \%)$. The patient demographics and tumor characteristics are summarized in Table 1. 
Table 1

Baseline characteristics

\begin{tabular}{|c|c|c|}
\hline & & $\begin{array}{l}\text { No patients } \\
(\%) \\
\mathrm{N}=79\end{array}$ \\
\hline \multirow[t]{2}{*}{ Sex } & Male & $72(91.1 \%)$ \\
\hline & Female & $7(8.9 \%)$ \\
\hline \multirow[t]{3}{*}{ Age } & Mean (ds) & $66.7(8.7)$ \\
\hline & $<70$ years & $50(63.3 \%)$ \\
\hline & $>70$ years & $29(36.7 \%)$ \\
\hline \multirow[t]{3}{*}{ ECOG } & $0-1$ & $62(78.5 \%)$ \\
\hline & 2 & $11(13.9 \%)$ \\
\hline & unknown & $6(7.6 \%)$ \\
\hline \multirow[t]{2}{*}{ Histology } & Squamous & $40(50.6 \%)$ \\
\hline & Non-squamous & $39(49.4 \%)$ \\
\hline \multirow[t]{3}{*}{ aPD-1/L1 drugs } & Nivolumab & $44(55.7 \%)$ \\
\hline & Atezolizumab & $20(25.3 \%)$ \\
\hline & Pembrolizumab & $15(19.0 \%)$ \\
\hline \multirow[t]{2}{*}{ Number of previous therapies } & 1 & $66(83.5 \%)$ \\
\hline & 2 & $13(16.5 \%)$ \\
\hline \multirow[t]{7}{*}{ Previous therapy } & Platinum+vinorelbine & $25(31.6 \%)$ \\
\hline & Platinum+pemetrexed & $24(30.4 \%)$ \\
\hline & Platinum+paclitaxel & $14(14.7 \%)$ \\
\hline & Platinum+gemcitabine & $7(8.9 \%)$ \\
\hline & Docetaxel monotherapy & $6(7.6 \%)$ \\
\hline & Paclitaxel monotherapy & $2(2.5 \%)$ \\
\hline & Bevacizumab+Paclitaxel+carboplatin & $1(1.3 \%)$ \\
\hline \multirow{3}{*}{$\begin{array}{l}\text { Time since initiation of platinum therapy. } \\
\text { months }\end{array}$} & Mean (ds) & $9.7(8.6)$ \\
\hline & $<6$ months & $34(43.0 \%)$ \\
\hline & $>6$ months & 45 (57.0\%) \\
\hline
\end{tabular}




\begin{tabular}{|c|c|c|}
\hline & & $\begin{array}{l}N^{\circ} \text { patients } \\
(\%)\end{array}$ \\
\hline & & $\mathrm{N}=79$ \\
\hline \multirow[t]{3}{*}{ Blood cell count. cell $/ \mu \mathrm{L}$} & White blood (ds) & $\begin{array}{l}9,300 \\
(3,900)\end{array}$ \\
\hline & Lymphocytes (ds) & $1,562(762)$ \\
\hline & Neutrophils (ds) & $\begin{array}{l}6,496 \\
(3,173)\end{array}$ \\
\hline \multirow[t]{2}{*}{ NLR } & $<5$ & $46(58.2 \%)$ \\
\hline & $\geq 5$ & $33(41.8 \%)$ \\
\hline \multirow[t]{2}{*}{$\mathrm{dNLR}$} & $<3$ & $51(64.6 \%)$ \\
\hline & $\geq 3$ & $28(35.4 \%)$ \\
\hline
\end{tabular}

Mean treatment duration with aPD1-i was 7.9 months (95\%Cl: $5.4-10.4)$ with a median of 4.1 months (95\%Cl: 2.3-5.8); the mean treatment duration for nivolumab, atezolizumab, and pembrolizumab was 5.5 months (95\% Cl: 3.0-8.0), 9.2 months ( $95 \% \mathrm{Cl}: 5.4-13.0)$, and 9.3 months (95\% Cl: 4.9-13.6), respectively. At the time of data collection, 18 patients $(22.8 \%)$ were still in treatment; the reasons for the discontinuation of aPD1-i therapy were disease progression, death, and adverse effects in 43 (54.4\%), 12 (15.2\%), and 6 (7.6\%) patients, respectively.

For the entire study population, the median OS and PFS were 7.3 months ( $95 \% \mathrm{Cl}: 3.4-11.3)$ and 4.4 months (95\% Cl: 2.3-6.5) months, respectively.

For the patients previously treated with G-CSF, the median OS was 15.8 months (95\% Cl: $1.2-30.3)$, whereas it was 5.3 months (95\% Cl: 2.3-8.3) in the patients not treated with G-CSF (HR $=0.63,95 \% \mathrm{Cl}$ : 0.32-1.25, p (log-rank $)=0.186)$. Median PFS was 6.1 months $(95 \% \mathrm{Cl}: 0.6-11.5)$ in the patients treated with G-CSF and 3.3 months $(95 \% \mathrm{Cl}: 1.7-4.8)$ in the patients not treated with G-CSF (HR $=0.75,95 \% \mathrm{Cl}$ : 0.40-1.38, $p$ (log-rank) $=0.352$ ) (Figure 1). Results for OS and PFS based on ECOG, NLR and dNLR are shown in Table 2. 
Table 2

Analysis of overall survival (OS) and progression-free survival (PFS) for Use of G-CSF, NLR and dNLR

\begin{tabular}{|c|c|c|c|c|}
\hline & os & & PFS & \\
\hline & $\begin{array}{l}\text { Median } \\
(95 \% \mathrm{Cl})\end{array}$ & hr $(95 \% \mathrm{Cl})$ & $\begin{array}{l}\text { Median } \\
(95 \% \mathrm{Cl})\end{array}$ & $\mathrm{hr}(95 \% \mathrm{Cl})$ \\
\hline $\begin{array}{l}\text { Yes G-CSF } \\
(n=19)\end{array}$ & $15.8(1.2-30.3)$ & \multirow[t]{2}{*}{$\begin{array}{l}0.63(0.32-1.25) \\
p=0.186\end{array}$} & $6.1(0.6-11.5)$ & $\begin{array}{l}0.75(0.40- \\
1.04)\end{array}$ \\
\hline No G-CSF $(n=60)$ & $5.3(2.3-8.3)$ & & $3.3(1.7-4.8)$ & $p=0.352$ \\
\hline$N L R<5(n=46)$ & $9.1(1.8-16.4)$ & \multirow[t]{2}{*}{$\begin{array}{l}1.42(0.80-2.51) \\
p=0.231\end{array}$} & $5.4(3.9-6.8)$ & $\begin{array}{l}1.45(0.85- \\
2.48)\end{array}$ \\
\hline$N L R \geq 5(n=33)$ & $4.3(2.8-5.7)$ & & $3.1(2.1-4.1)$ & $P=0.176$ \\
\hline $\mathrm{dNLR}<3 \quad(\mathrm{n}=51)$ & $13.9(5.4-22.4)$ & \multirow{2}{*}{$\begin{array}{l}1.83(1.03-3.25) \\
p=0.040\end{array}$} & $5.4(1.7-4.5)$ & $\begin{array}{l}1.28(0.73- \\
2.22)\end{array}$ \\
\hline$d N L R \geq 3(n=28)$ & $3.6(2.4-4.8)$ & & $3.1(2.3-6.5)$ & $p=0.388$ \\
\hline
\end{tabular}

The effectiveness of G-CSF on the OS of the different subgroups is shown in Table 3 and Figure 2. 
Table 3

Analysis of overall survival by subgroups

\begin{tabular}{|lll|}
\hline & \multicolumn{1}{c}{ Yes Use G-CSF } & No Use G-CSF \\
\cline { 2 - 3 } & Median (95\%Cl) & Median (95\%Cl) \\
\hline Population total $(n=79)$ & $\mathbf{1 5 . 8}(1.2-30.3)$ & $\mathbf{5 . 3}(2.3-8.3)$ \\
\hline NLR<5 $(n=46)$ & $19.8(0-40.2)$ & $8.2(0-16.6)$ \\
\hline NLR $\geq 5(n=33)$ & $15.8(0-41.9)$ & $3.6(2.5-4.7)$ \\
\hline dNLR<3 $(n=51)$ & $19.8(3.7-35.9)$ & $11.3(0-22.8)$ \\
\hline dNLR $\geq 3(n=28)$ & $15.8(0-34.8)$ & $3.6(2-5.1)$ \\
\hline ECOG=0-1 (n=62) & $19.8(8.2-31.4)$ & $11.3(3.9-18.7)$ \\
\hline ECOG=2 (n=11) & $2.9(--)$ & $1.1(0.9-1.3)$ \\
\hline Squamous $(n=40)$ & $15.8(0.9-30.6)$ & $4.4(1.8-7)$ \\
\hline Nonsquamous $(n=39)$ & $38.5(--)$ & $5.4(1.6-9.2)$ \\
\hline TiPt $<6$ months $(n=33)$ & $3(0-18.3)$ & $2.6(0.9-4.4)$ \\
\hline TiPt $\geq 6$ months $(n=46)$ & $38.5(--)$ & $15.7(2.4-29)$ \\
\hline NIV $(n=44)$ & $8.7(2.1-15.2)$ & $3.6(2.6-4.6)$ \\
\hline ATE $(n=20)$ & $15.7(--)$ & $-(-)$ \\
\hline PEM $(n=15)$ & $19.8(--)$ & $11.3(0-29.3)$ \\
\hline
\end{tabular}

\section{Discussion}

We hypothesized that the use of G-CSF in nSCLC patients could decrease OS because a possible increase in the neutrophil count would reduce the survival rate by increasing the NLR and the dNLR. However, the results of this study showed higher OS in the group of patients who received G-CSF supportive therapy prior to the treatment with aPD1-i in comparison to that of the group that did not receive G-CSF (HR = $0.63,95 \% \mathrm{Cl}$ : $0.32-1.25, \mathrm{p}$ (log-rank) $=0.186)$. This difference in OS was not statistically significant, but it was observed in all the subgroups (Figure 2).

OS prognostic factors for aPD1-i drugs in nSCLC and other cancer can be classified into factors dependent on the patient, tumor, drugs, and environment; the patient-dependent prognostic factors include NLR and dNLR. Patients with NLR $\geq 5$ or dNLR $\geq 3$ have a lower OS (20),(21). Thus, patients with an elevated neutrophil count may have a poor response to aPD1-i drugs. In contrast, patients with NLR $<5$ or $\mathrm{dNLR}<3$ have better OS. In our population, the prognostic value of NLR and dNLR can be observed in the OS (Table 2). The median OS was higher and statistically significant in the case of patients with a $d N L R<3$. For patients with a NLR $<5$, the OS was higher without reaching statistical significance. 
In the group of patients who received G-CSF, the median OS was similar in the subgroups with NLR $\geq 5$ and NLR $<5$ (Table 3). A similar result was achieved in the subgroup of patients with dNLR $\geq 3$, as its median OS was like that in the subgroup with $\mathrm{dNLR}<3$. These results show an improvement in the prognosis of nSCLC when G-CSF is used in a group of patients with a priori poor prognosis, i.e., patients with NLR $\geq 5$ or $\mathrm{dNLR} \geq 3$.

The improvement in prognosis with the use of G-CSF was observed in all the subgroups in terms of histology, aPD1-i drug performance status, and time elapsed since the initiation of platinum therapy until aPD1-i drug administration. However, conclusions cannot be drawn because of the difference in the number of cases in each subgroup.

The results of this study are difficult to interpret because previous studies have reported that the use of GCSF increases the neutrophil count, NLR and dNLR, therefore, causes a worse prognosis (19), (20), (21). The role of neutrophils in antitumor immunity remains unclear. Data indicate that the tumor microenvironment induces a deterioration in antitumor immunity through modulation of PD-L1 expression in tumor-infiltrating neutrophils. It has also been shown in vitro that granulocyte-macrophage colony-stimulating factor (GM-CSF) can increase the expression of PD-L1 in neutrophils of the tumor microenvironment thus suppressing the proliferation and activation of T cells (23).

On the other hand, there are data that show that with the use of G-CSF, the significant increase in absolute numbers of lymphocytes, monocytes, CD3+, CD4+, and CD8+ T cells, and the ratios of CD4/CD8 and dendritic cells in peripheral blood grafts is higher than in bone marrow grafts (24) which causes a faster immune reconstitution in the receptors of blood stem cell transplants; they have higher lymphocyte subset count, which results in fewer infections (25). These data indicate a beneficial effect of G-CSF administration on the antitumor immunity of lymphocytes.

The population included in this study was previously treated for lung cancer and received G-CSF prior to treatment with the aPD1-i drug. The regimens used in the first-line therapy for nSCLC are classified as intermediate risk for febrile neutropenia (incidence 10-20\%); therefore, prophylactic use of G-CSF would be considered in the first chemotherapy cycle in case of the existence of associated risk factors for neutropenia and after chemotherapy cycle if dose-limiting neutropenic event or febrile neutropenia (22). In our study, $24.1 \%$ of the patients received G-CSF for febrile neutropenia, a value slightly higher than the $20 \%$ expected incidence for febrile neutropenia. With the results of incidence of febrile neutropenia and the OS for patients who received G-CSF, obtained in this study, use since the first cycle of chemotherapy in the first line of nSCLC could be considered.

Currently, first-line treatment for nSCLC includes the combination of aPD1-i plus chemotherapy. In this situation, G-CSF could also be used from the first cycle of treatment as prophylaxis for febrile neutropenia.

Therefore, in this study, we elucidate the effectiveness of G-CSF during chemotherapy prior to treatment with aPD1-i and associated with combination regimens of aPD1-i plus chemotherapy for first-line 
treatment of nSCLC. The use of G-CSF as primary prophylaxis of febrile neutropenia, in these situations, could be considered as high-risk neutropenia chemotherapy regimens. This approach should be validated in larger studies using real world data or prospective studies should be conducted based on this hypothesis.

\section{Conclusion And Relevance}

This study showed a trend toward higher OS in patients who received the supportive G-CSF therapy compared to those who did not receive it; this trend was observed in all the subgroups. The use of G-CSF is a good prognostic factor for the survival of a patient undergoing aPD1-i therapy and represent a change in the guidelines for prophylaxis of chemotherapy-induced neutropenia and the use of hematopoietic factors.

\section{Declarations}

\section{Statements and Declarations}

The authors declare no potential conflicts of interest with respect to the research, Authorship, and/or publication of this article.

This work was presented as abstract at 2020 ASCO Annual Meeting

\section{Funding}

The authors declare that they have not received any financial support for the research, authorship, and/or publication of this article

\section{Individual Authors' Contribution}

\begin{tabular}{|llllll|}
\hline & $\begin{array}{l}\text { Study } \\
\text { Design }\end{array}$ & $\begin{array}{l}\text { Data } \\
\text { Collect }\end{array}$ & $\begin{array}{l}\text { Statistical } \\
\text { analysis }\end{array}$ & $\begin{array}{l}\text { Drafting } \\
\text { manuscript }\end{array}$ & $\begin{array}{l}\text { Final } \\
\text { revision }\end{array}$ \\
\hline Juan F. Marín Pozo & Yes & Yes & Yes & Yes & Yes \\
\hline Carmen L. Muñoz Cid & Yes & Yes & Yes & Yes & Yes \\
\hline $\begin{array}{l}\text { Raquel Claramunt } \\
\text { García }\end{array}$ & - & Yes & - & Yes & Yes \\
\hline Elvira Marín Caba & - & Yes & - & Yes & Yes \\
\hline $\begin{array}{l}\text { Ana Laura Ortega } \\
\text { Granados }\end{array}$ & Yes & Yes & - & Yes & Yes \\
\hline
\end{tabular}

\section{Ethics approval}


This study was performed in line with the principles of the declaration of Helsinki. Approval was granted by the of Ethics Committee of investigation (CEIm) of Province of Jaen ((Date: 30/05/2019).

\section{Consent to participate}

Informed consent was obtained (or no refusal to participate in studies when it could not be obtained) from all individual participants included in the study.

\section{Consent to publish}

This manuscript does not contain individual personal data

\section{References}

1. NCCN Clinical Practice Guidelines in Oncology (NCCN Guidelines ${ }^{\circledR}$ ). Non-Small Cell Lung Cancer. Version 4.2021 - March 3, 2021 [Internet]. [citado 9 de junio de 2021]. Disponible en: https://www.nccn.org/professionals/physician_gls/pdf/nscl.pdf.

2. Callahan MK, Postow MA, Wolchok JD. Targeting T Cell Co-receptors for Cancer Therapy. Immunity. 2016;44(5):1069-78.

3. Brahmer J, Reckamp KL, Baas P, et al. Nivolumab versus Docetaxel in Advanced Squamous-Cell Non-Small-Cell Lung Cancer. N Engl J Med. 2015;373(2):123-35.

4. Borghaei H, Paz-Ares L, Horn L, et al. Nivolumab versus Docetaxel in Advanced Nonsquamous NonSmall-Cell Lung Cancer. N Engl J Med. 2015;373(17):1627-39.

5. Herbst RS, Baas P, Kim D-W, et al. Pembrolizumab versus docetaxel for previously treated, PD-L1positive, advanced non-small-cell lung cancer (KEYNOTE-010): a randomised controlled trial. Lancet Lond Engl. 2016;387(10027):1540-50.

6. Rittmeyer A, Barlesi F, Waterkamp D, et al. Atezolizumab versus docetaxel in patients with previously treated non-small-cell lung cancer (OAK): a phase 3 , open-label, multicentre randomised controlled trial. Lancet Lond Engl. 2017;389(10066):255-65.

7. Oya $\mathrm{Y}$, Yoshida $\mathrm{T}$, Kuroda $\mathrm{H}$, et al. Predictive clinical parameters for the response of nivolumab in pretreated advanced non-small-cell lung cancer. Oncotarget 7 de octubre de. 2017;8(61):103117-28.

8. Gibney GT, Weiner LM, Atkins MB. Predictive biomarkers for checkpoint inhibitor-based immunotherapy. Lancet Oncol. 2016;17(12):e542-51.

9. Rochet NM, Kottschade LA, Grotz TE, Porrata LF, Markovic SN. The Prognostic Role of the Preoperative Absolute Lymphocyte Count and Absolute Monocyte Count in Patients With Resected Advanced Melanoma. Am J Clin Oncol. 2015;38(3):252-8.

10. Kumagai $S$, Marumo S, Shoji T, et al. Prognostic impact of preoperative monocyte counts in patients with resected lung adenocarcinoma. Lung Cancer Amst Neth. 2014;85(3):457-64.

11. Pine JK, Morris E, Hutchins GG, et al. Systemic neutrophil-to-lymphocyte ratio in colorectal cancer: the relationship to patient survival, tumour biology and local lymphocytic response to tumour. $\mathrm{Br} \mathrm{J}$ 
Cancer. 2015;113(2):204-11.

12. Moses K, Brandau S. Human neutrophils: Their role in cancer and relation to myeloid-derived suppressor cells. Semin Immunol. 2016;28(2):187-96.

13. Tang H, Lu W, Li B, Li C, Xu Y, Dong J. Prognostic significance of neutrophil-to-lymphocyte ratio in biliary tract cancers: a systematic review and meta-analysis. Oncotarget. 2017;8(22):36857-68.

14. Çalışkan S, Sungur M, Kaba S, Özsoy E, Koca O, Öztürk M. Neutrophil-to-Lymphocyte Ratio in Renal Cell Carcinoma Patients. Folia Med (Plovdiv). 2018;60(4):553-7.

15. Templeton AJ, McNamara MG, Šeruga B, et al. Prognostic role of neutrophil-to-lymphocyte ratio in solid tumors: a systematic review and meta-analysis. J Natl Cancer Inst. 2014;106(6):dju124.

16. Zucker A, Winter A, Lumley D, Karwowski P, Jung M-K, Kao J. Prognostic role of baseline neutrophilto-lymphocyte ratio in metastatic solid tumors. Mol Clin Oncol. 2020;13(4):25.

17. Ferrucci PF, Ascierto PA, Pigozzo J, et al. Baseline neutrophils and derived neutrophil-to-lymphocyte ratio: prognostic relevance in metastatic melanoma patients receiving ipilimumab. Ann Oncol. 2016;27(4):732-8.

18. Capone M, Giannarelli D, Mallardo D, et al. Baseline neutrophil-to-lymphocyte ratio (NLR) and derived NLR could predict overall survival in patients with advanced melanoma treated with nivolumab. J Immunother Cancer. 2018;6(1):74.

19. Jin J, Yang L, Liu D, Li W. Association of the neutrophil to lymphocyte ratio and clinical outcomes in patients with lung cancer receiving immunotherapy: a meta-analysis. BMJ Open. 2020;10(6):e035031.

20. Saravia D, Okabe N, Park W, et al. Neutrophil-lymphocyte-ratio to complement the prediction ability of PD-L1 expression for outcomes in patients with advanced non-small cell lung cancer treated with PD1/PD-L1 inhibitors. J Clin Oncol. 2018;36(15_suppl):e15102-2.

21. Mezquita L, Arbour K, Auclin E, et al. 1407P - Derived neutrophil-to lymphocyte ratio (dNLR) change between baseline and cycle 2 is correlated with benefit during immune checkpoint inhibitors (ICI) in advanced non-small cell lung cancer (NSCLC) patients. Ann Oncol. 2018;29:viii508.

22. NCCN Clinical Practice Guidelines in Oncology (NCCN Guidelines®). Hematopoietic Growth Factors. Version 4.2021 - May 20, 2021. [Internet]. [citado 9 de junio de 2021]. Disponible en: https://www.nccn.org/professionals/physician_gls/pdf/growthfactors.pdf.

23. He G, Zhang H, Zhou J, et al. Peritumoural neutrophils negatively regulate adaptive immunity via the PD-L1/PD-1 signalling pathway in hepatocellular carcinoma. J Exp Clin Cancer Res CR [Internet]. 18 de noviembre de 2015 [citado 27 de mayo de 2021];34. Disponible en: https://www.ncbi.nlm.nih.gov/pmc/articles/PMC4652417/.

24. Zhao X-Y, Chang Y-J, Huang X-J. [Effects of rhG-CSF mobilization on immunological properties of grafts from peripheral blood and bone marrow]. Zhongguo Shi Yan Xue Ye Xue Za Zhi agosto de. 2006;14(4):787-90.

25. Storek J, Dawson MA, Storer B, et al. Immune reconstitution after allogeneic marrow transplantation compared with blood stem cell transplantation. Blood 1 de junio de. 2001;97(11):3380-9. 
Figures

A

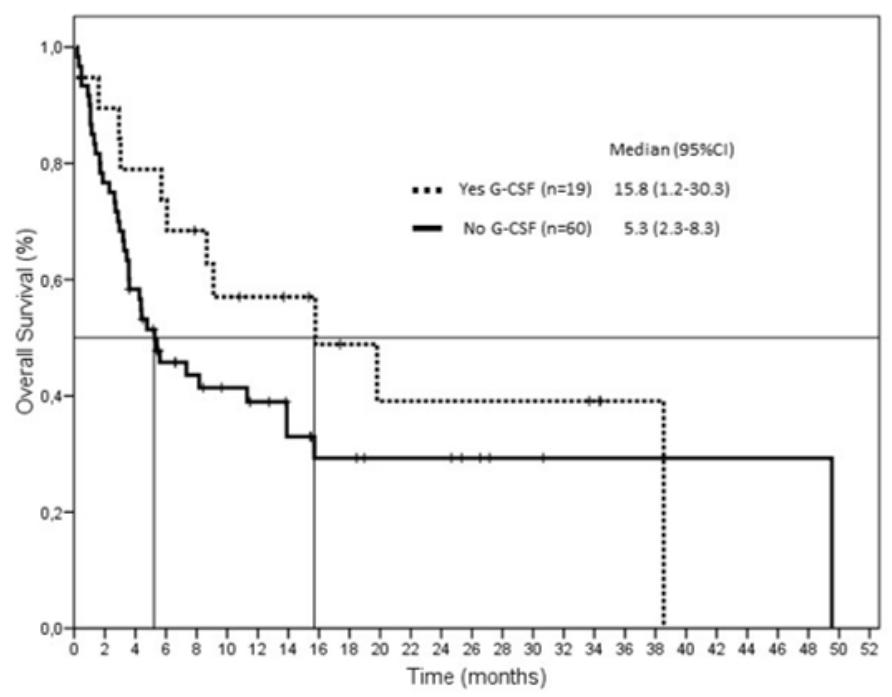

B

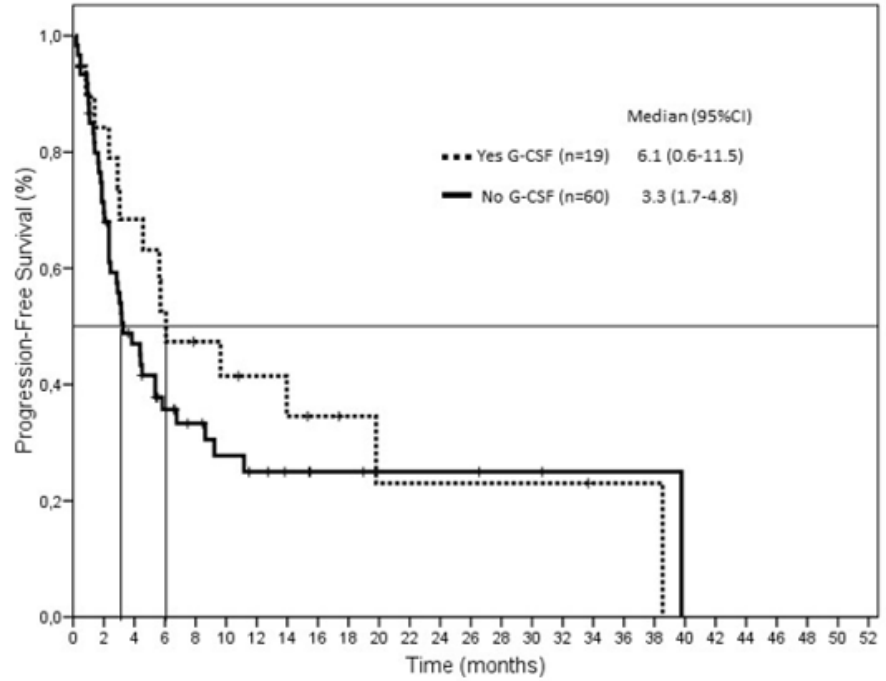

\section{Figure 1}

Kaplan-Meier analysis of overall survival (figure 1-A) and progression-free survival (figure 1-B), for all patients

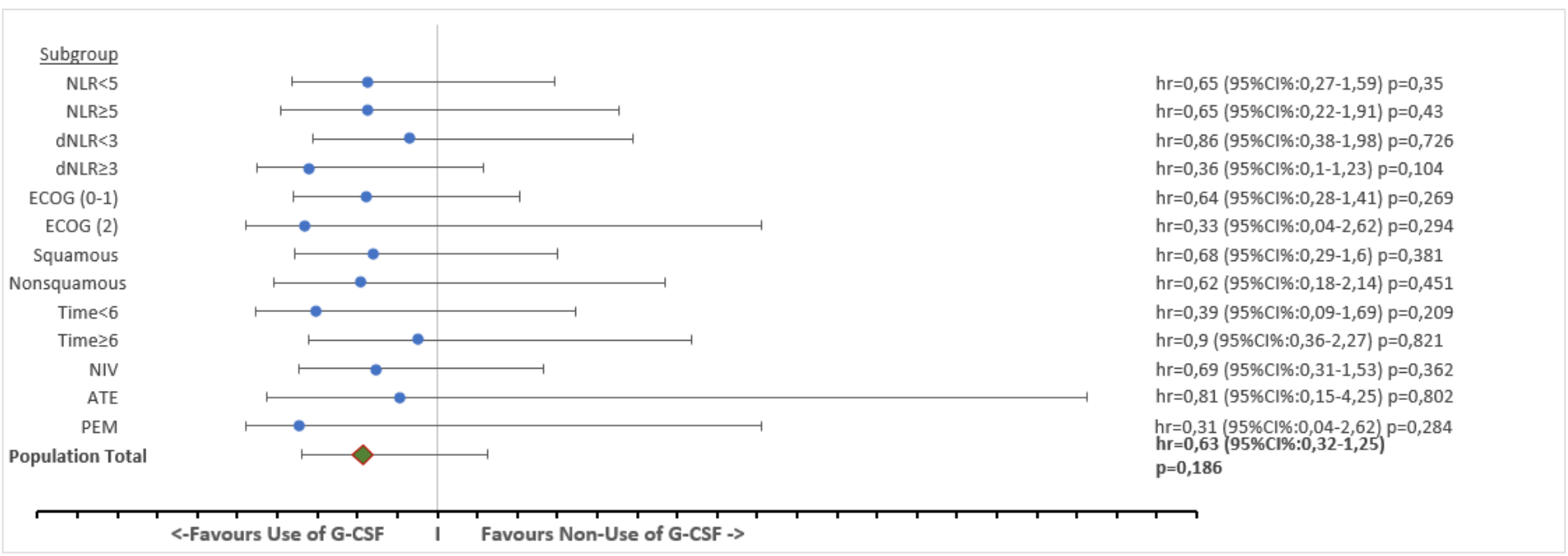

\section{Figure 2}

Subgroup analysis of overall survival 